CLINICAL STUDY

\title{
Inositol administration reduces oxidative stress in erythrocytes of patients with polycystic ovary syndrome
}

\author{
Gabriella Donà, Chiara Sabbadin ${ }^{1}$, Cristina Fiore ${ }^{1}$, Marcantonio Bragadin ${ }^{2}$, Francesco L Giorgino ${ }^{3}$, \\ Eugenio Ragazzi ${ }^{4}$, Giulio Clari, Luciana Bordin* and Decio Armanini ${ }^{1, *}$ \\ Department of Biological Chemistry, University of Padua, Viale G. Colombo 3, 35131 Padua, Italy, ${ }^{1}$ Endocrinology Unit, Department of Medical and \\ Surgical Sciences, University of Padua, Via Ospedale 105, 35128 Padua, Italy, ${ }^{2}$ Department of Environmental Sciences, University of Venice, Venice, Italy, \\ ${ }^{3}$ Associazione Ginecologi Extra Ospedalieri (A.G.E.O.), Padua, Italy and ${ }^{4}$ Department of Pharmacology and Anesthesiology, University of Padua, Padua, \\ Italy
}

(Correspondence should be addressed to L Bordin; Email: luciana.bordin@unipd.it; D Armanini; Email: decio.armanini@unipd.it)

*(L Bordin and D Armanini contributed equally to this work)

\begin{abstract}
Objective: Possibly due to a deficiency of insulin mediators, polycystic ovary syndrome (PCOS) is often associated with insulin resistance (IR) and hyperinsulinemia, likely responsible for an elevated production of reactive oxygen species. We investigated oxidative-related alterations in erythrocytes and anti-inflammatory effects of inositol in women with PCOS before and after treatment with myo-inositol (MYO).

Methods: Twenty-six normal-weight PCOS patients were investigated before and after MYO administration ( $1200 \mathrm{mg}$ /day for 12 weeks; $n=18)$ or placebo $(n=8)$ by evaluating serum testosterone, serum androstenedione, fasting serum insulin, fasting serum glucose, insulin area under the curve (AUC), and glucose AUC after oral glucose tolerance test and homeostasis model of assessment-IR. In erythrocytes, band 3 tyrosine phosphorylation (Tyr-P) level, glutathione (GSH) content, and glutathionylated proteins (GSSP) were also assessed.

Results: Data show that PCOS patients' erythrocytes underwent oxidative stress as indicated by band 3 Tyr-P values, reduced cytosolic GSH content, and increased membrane protein glutathionylation. MYO treatment significantly improved metabolic and biochemical parameters. Significant reductions were found in IR and serum values of androstenedione and testosterone. A significant association between band 3 Tyr-P levels and insulin AUC was found at baseline but disappeared after MYO treatment, while a correlation between band 3 Tyr-P and testosterone levels was detected both before and after MYO treatment.

Conclusions: PCOS patients suffer from a systemic inflammatory status that induces erythrocyte membrane alterations. Treatment with MYO is effective in reducing hormonal, metabolic, and oxidative abnormalities in PCOS patients by improving IR.
\end{abstract}

European Journal of Endocrinology 166 703-710

\section{Introduction}

Polycystic ovary syndrome (PCOS) is one of the most common endocrine-metabolic diseases, affecting $6-10 \%$ of women of reproductive age (1). It is characterized by hyperandrogenism, hirsutism, and oligo- or anovulation. Increasing evidence supports the central role of insulin resistance (IR) and compensatory hyperinsulinemia in the pathogenesis of the syndrome (2) and in patients' increased risk of developing dyslipidemia, hypertension, impaired glucose tolerance, type 2 diabetes mellitus, and cardiovascular disease (3). Some actions of insulin may involve low-molecular weight inositol phosphoglycan (IPG) mediators (also known as putative insulin mediators or second messengers) $(4,5)$ and several pieces of evidence suggest that a deficiency in a specific D-chyro inositol (DCI)-containing IPG $(6,7,8)$ and/or altered DCI metabolism may contribute to IR $(9,10)$. Previous studies have shown that oral administration of DCI to women with PCOS increases the action of insulin, improving ovulatory function and decreasing blood pressure, and serum androgen and plasma triglyceride concentrations $(9,10)$.

Oxidative stress (OS) is involved in the pathogenesis and future complications of PCOS. This condition occurs when reactive oxygen species (ROS), which are intermediaries of a normal oxygen metabolism, are produced faster than the endogenous antioxidant defense systems can neutralize. Previous studies have demonstrated that hyperglycemia increases ROS generation from peripheral blood leukocytes (11). 
The resulting OS may contribute to a pro-inflammatory state that induces IR and hyperandrogenism in women with this disorder (12) and also increases the risk of cardiovascular disease (13).

Many proteins are susceptible to attack by ROS, especially as sulfhydryl groups are among the most easily oxidized protein residues. Oxidation can lead to inter- and/or intramolecular cross-linking, thus inducing protein degradation $(14,15)$, clustering $(16,17$, $18)$, and enzyme inactivation $(18,19)$.

We recently demonstrated that diamide-treated erythrocytes express a well-defined tyrosine phosphorylation (Tyr-P) level of membrane proteins, particularly of band 3 (20). Alterations in band 3 Tyr-P levels represent preexisting modifications of membrane status, like that observed in glucose-6-phosphate dehydrogenase (G6PD)-deficient patients $(18,21)$ suffering from chronic impairment of antioxidant defenses or in endometriotic women (22) with systemic inflammation. Diamide, a mild oxidant, affects the sulfhydryl groups of cysteines by inducing disulfide bond formation and triggers band 3 Tyr-P $(16,17,18,20,21,22)$, which is a useful parameter in the evaluation of oxidation-related damage to cells $(18,21,22)$. We also observed that, while diamide treatment reduced total glutathione (GSH) contents detectable in the cytosol of endometriotic patients, normal subjects were unaffected, and the differences between GSH content before and after diamide treatment were defined as $\Delta \mathrm{GSH}$, an additional parameter of inflammatory status in endometriosis (22).

The aim of this study was to evaluate any effects on clinical, hormonal, and glucose metabolism parameters in PCOS patients before and after 12 weeks of inositol dietary supplement. We used myo-inositol (MYO) because it is widely distributed in nature (23) and commercially available, whereas DCI, the product of oxidoreductive epimerization of the carbon 1 hydroxyl group of MYO, is relatively rare and present in plants and seeds (24).

In addition, in order to evaluate the inositol effects on erythrocyte membrane functionality and cell redox status, we determined diamide-related band 3 Tyr-P levels and contents of both GSH and membrane glutathionylated proteins in patients' erythrocytes.

\section{Materials and methods}

\section{Materials}

Reagents were purchased from Sigma, and antiphosphotyrosine (P-Tyr; PY20) mouse MAB was obtained from Biosource-Invitrogen (Camarillo, CA, USA). The protease inhibitor cocktail and anti-actin mouse MAB came from Calbiochem (Darmstadt, Germany). Antimouse secondary antibody conjugated with HRP came from Bio-Rad Laboratories. MYO was purchased from Progine (Firenze, Italy).
Testosterone was measured by electrochemiluminescence immunoassay (ECLIA) using commercially available kits (Roche Diagnostics $\mathrm{GmbH}$ ); 17-OH progesterone was measured by a solid-phase ELISA using commercially available kits (DRG Instruments GmbH, Marburg, Germany); androstenedione was measured by a solid-phase, competitive chemiluminescent enzyme immunoassay using commercially available kits (Siemens Healthcare Diagnostics Products Ltd., Llanberis, Gwynedd, UK). The intra-assay and interassay coefficients of variation were $<9 \%$.

Serum glucose levels were measured enzymatically with glucose hexokinase kit (Roche Diagnostics), and serum insulin levels were measured by ECLIA (Roche) with Immulite 2000 DPC Siemens Analyzer.

\section{Study protocol}

Twenty-six women with PCOS, between the ages of 22 and 30 years, were enrolled at the Department of Medical and Surgical Sciences of the University of Padova, Italy. Diagnosis of PCOS was defined when two of the following criteria were fulfilled: oligo- and/or anovulation $(\leq 8$ menstrual periods in the previous year), clinical and/or biochemical signs of hyperandrogenism, polycystic ovaries, and exclusion of other etiologies (25). Exclusion criteria included pregnancy, body mass index (BMI) $>25 \mathrm{~kg} / \mathrm{m}^{2}$, hyperprolactinemia, thyroid dysfunction, Cushing's syndrome, and late-onset adrenal hyperplasia. None of the women had diabetes or had taken oral contraceptives, antiinflammatory drugs, or other hormonal drugs during the previous 3 months. This study was approved by the Ethics Committee for Research and Clinical Trials of our University and all the patients gave their written informed consent.

At the time of entry into the study, all patients had serum progesterone concentrations below $2.5 \mathrm{ng} / \mathrm{ml}$. On the first day of the study (baseline, $\mathrm{T}_{0}$ ), after a $12 \mathrm{~h}$ overnight fast, blood samples were collected to measure serum testosterone, androstenedione, glucose, and insulin.

A citrated blood sample was collected from each subject to evaluate Tyr-P levels and assess GSH and glutathionylated proteins (GSSP).

A $2 \mathrm{~h}$ oral glucose tolerance test (OGTT) with $75 \mathrm{~g}$ glucose was carried out. Blood samples were collected after $15,30,60,90$, and 120 min to determine serum glucose and insulin; values are expressed as the area under the curve (AUC) of glucose and insulin. Index of IR was a peak of serum insulin value $>70 \mathrm{mU} / \mathrm{l}$ during OGTT.

Homeostasis model of assessment-IR (HOMA-IR) was also used, according to (26). During the same examination, anthropometric (weight, height, and BMI) and clinical (Ferriman-Gallwey score, heart rate, and systolic and diastolic blood pressure) measurements were taken for all subjects. 
After baseline evaluations, women were randomized to receive MYO dietary supplement powder $1200 \mathrm{mg} /$ day $(n=18)$ or matched placebo powder $(n=8)$ for 12 weeks. To avoid selection bias, patients were randomized to each group (treatment or placebo) using a randomly generated list with the Excel 'Rand()' function. In the MYO group, a larger number of patients were set 'a priori' in order to be more confident with the results and to avoid any possible distortion due to the variability in pharmacokinetics of the substance and to the variable ranges of dietary inositol assumption.

Administration was from predosed sachets containing the daily dose; the powder was dissolved in water before oral intake once a day. Subjects were instructed not to change their eating habits, activity level, or lifestyle during the study. At the end of the study, the measurements and tests performed at baseline were repeated (posttreatment time, $\mathrm{T}_{1}$ ).

\section{Treatment of erythrocytes}

Red blood cells (RBC) were pelleted at $750 \boldsymbol{g}$ for $3 \mathrm{~min}$. After removal of supernatant, packed RBC were washed three times at $750 \boldsymbol{g}$ for $3 \mathrm{~min}$ in five volumes of Dulbecco's PBS (D-PBS) to avoid contamination by leucocytes and platelets. Packed cells $(50 \mu \mathrm{l})$ were resuspended (at 20\% hematocrit) in D-PBS and treated at $35^{\circ} \mathrm{C}$ for $30 \mathrm{~min}$ in the absence (Basal) or presence (Diamide) of $1.5 \mathrm{mM}$ diamide (dissolved in D-PBS) (22).

Samples were then centrifuged and packed cells were washed in D-PBS, and they underwent hemolysis in $1.5 \mathrm{ml}$ hypotonic buffer ( $5 \mathrm{mM}$ sodium phosphate, $\mathrm{pH}$ $8,0.02 \%$ sodium azide $\left(\mathrm{NaN}_{3}\right), 30 \mu \mathrm{M}$ phenylmethylsulfonyl fluoride, $1 \mathrm{mM}$ sodium orthovanadate, and a protease inhibitor cocktail).

Membranes were separated from the cytosol by centrifugation (16 $100 \boldsymbol{g}$ for $20 \mathrm{~min}$ in an Eppendorf centrifuge) and washed once in hypotonic buffer. Aliquots of membranes $(10 \mu \mathrm{g})$ were suspected to SDS-PAGE (10\% gels), transferred to nitrocellulose membranes, and immunostained first with anti-P-Tyr antibody and then with anti-actin sample loading controls.

Band 3 Tyr-P levels were evaluated densitometrically. The Tyr-P value of diamide-treated erythrocytes from PCOS patients before $\left(\mathrm{T}_{0}\right)$ and after $\left(\mathrm{T}_{1}\right) \mathrm{MYO} /$ placebo treatment was calculated as the ratio to the Tyr-P level of diamide-treated erythrocytes obtained in healthy controls (chosen as arbitrary comparison unit, experimentally determined as $0.98 \pm 0.065$, mean value \pm s.D.). To express a standardized measure of Tyr-P variation after treatment (defined as the Tyr-P variation index, $\left.V_{i(T y r-P)}\right)$ with respect to baseline value, the following formula was used:

$$
V_{i(\mathrm{Tyr}-\mathrm{P})}=1-\left(\frac{\mathrm{Tyr}-\mathrm{P}_{T_{1}}}{\mathrm{Tyr}-\mathrm{P}_{T_{0}}}\right) .
$$

\section{Determination of GSH and GSSP in erythrocytes}

Total GSH was determined according to the Tietze method (27). Briefly, $10 \mu \mathrm{l}$ cytosol obtained from differently treated erythrocytes were added to $2 \mathrm{ml}$ reaction mixture containing $1.9 \mathrm{ml}$ phosphate

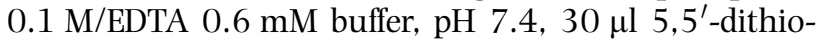
bis(2-nitrobenzoic acid) $10 \mathrm{mM}, 100 \mu \mathrm{l}$ NADPH $5 \mathrm{mM}$, and $10 \mu \mathrm{g}$ glutathione reductase and analyzed spectrophotometrically at $412 \mathrm{~nm}$. The decrease in total GSH content after diamide treatment $(\Delta \mathrm{GSH})$ was expressed as $1-\mathrm{GSH}_{\text {(Diamide) }} / \mathrm{GSH}_{\text {(Basal) }}(22)$.

A variation index for $\Delta G S H\left(V_{i(\Delta G S H)}\right)$ was calculated, as for Tyr-P, according to the following formula:

$V_{i(\Delta \mathrm{GSH})}=1-\left(\frac{\Delta \mathrm{GSH}_{T_{1}}}{\Delta \mathrm{GSH}_{T_{0}}}\right)$.

GSSP was determined according to (28). Briefly, membranes, obtained as described above, were deproteinized by adding trichloroacetic acid (TCA; 5\% final concentration). After centrifugation for $3 \mathrm{~min}$, a $16100 \mathrm{~g}$ acid-precipitated membrane protein preparation was resuspended and brought to $\mathrm{pH} 12$, which induces GSH release from proteins via a cysteine-reduced/oxidized (SH/SS) exchange. The reaction was stopped by adding TCA (5\% final concentration); the amount of released GSH was determined enzymatically in the supernatants of centrifuged samples.

The increase in GSH released from membranes of erythrocytes treated with diamide, which was representative of the increase in glutathionylated protein content $(\Delta \mathrm{GSSP})$, was expressed as $\left(\mathrm{GSH}_{\text {(Diamide) }} /\right.$ $\left.\mathrm{GSH}_{(\text {Basal })}\right)-1$.

Also here, a variation index for $\Delta \mathrm{GSSP}\left(\mathrm{V}_{\mathrm{i}(\Delta \mathrm{GSSP})}\right)$ was calculated, according to the following formula:

$$
V_{i(\Delta \mathrm{GSSP})}=1-\left(\frac{\Delta \mathrm{GSSP}_{T_{1}}}{\Delta \mathrm{GSSP}_{T_{0}}}\right) .
$$

\section{Statistical analysis}

Data are expressed as mean \pm s.D. Comparisons were obtained with Student's $t$-test for paired or unpaired data, as appropriate. Statistical significance was set at $P<0.05$ (two tailed). Any relationships between pairs of variables were tested by least-squares linear regression. Pearson's correlation coefficient $r$ was used to quantify the strength of the relationships. The statistical significance of $r$ was determined by ANOVA; a $P$ value of $<0.05$ was considered statistically significant (two tailed). Comparison between regression lines was performed with ANOVA on slope and intercept parameters. 
Table 1 Baseline clinical characteristics and hematochemical parameters of two groups of PCOS patients. Data are mean \pm s.D. Normal values for serum testosterone and androstenedione and insulin and glucose AUC were calculated in a group of healthy women $(n=15)$ : average age, 23.6 \pm 1.5 ; weight, $57.9 \pm 4.6$; and BMI, 21.4 \pm 1.2 .

\begin{tabular}{|c|c|c|c|c|}
\hline Parameters & Normal range & MYO group $(n=18)$ & Placebo group $(n=8)$ & $\boldsymbol{P}^{\mathrm{a}}$ \\
\hline Age (years) & - & $23.5 \pm 2.1$ & $23.6 \pm 1.4$ & 6.1257 \\
\hline Weight $(\mathrm{kg})$ & - & $58.9 \pm 6.4$ & $58.8 \pm 3.5$ & 6.6347 \\
\hline $\operatorname{BMI}\left(\mathrm{kg} / \mathrm{m}^{2}\right)$ & - & $21.6 \pm 1.9$ & $21.9 \pm 0.6$ & 4.3354 \\
\hline \multicolumn{5}{|l|}{ Blood pressure $(\mathrm{mmHg})$} \\
\hline Systolic & $<120$ & $117.2 \pm 10.7$ & $112.5 \pm 7.1$ & 1.8653 \\
\hline Diastolic & $<80$ & $72.2 \pm 4.3$ & $71.3 \pm 3.5$ & 4.0257 \\
\hline Serum testosterone $(\mathrm{nmol} / \mathrm{l})$ & $0-8 \pm 0-2$ & $1.77 \pm 0.50$ & $1.96 \pm 0.25$ & 2.1701 \\
\hline Serum androstenedione $(\mathrm{nmol} / \mathrm{l})$ & $7.5 \pm 1-9$ & $14.99 \pm 3.51$ & $14.34 \pm 2.13$ & 4.3972 \\
\hline Fasting serum insulin $(\mathrm{mU} / \mathrm{l})$ & $5-20$ & $7.49 \pm 4.44$ & $6.75 \pm 1.67$ & 4.5354 \\
\hline Fasting serum glucose $(\mathrm{mmol} / \mathrm{l})$ & $3.7-6.0$ & $4.79 \pm 0.32$ & $4.73 \pm 0.38$ & 4.5910 \\
\hline Insulin AUC (mU/l per min) & $2467.34 \pm 654.45$ & $6989.47 \pm 2301.69$ & $7499.38 \pm 2047.60$ & 4.1354 \\
\hline Glucose AUC ( $\mathrm{mmol} / \mathrm{l}$ per $\mathrm{min})$ & $729.23 \pm 37.25$ & $712.06 \pm 76.71$ & $700.12 \pm 46.61$ & 4.7840 \\
\hline HOMA-IR & $<2.5$ & $1.60 \pm 0.99$ & $1.40 \pm 0.40$ & 4.0625 \\
\hline
\end{tabular}

aStatistical analysis was obtained with Student's $t$-test between the two groups.

\section{Results}

Baseline clinical characteristics and hematochemical parameters determined in the two groups of PCOS showed no significant differences (Table 1).

Following the 12-week treatment, the studied parameters in the MYO group showed significant decreases with respect to baseline (Table 2). Conversely, in the placebo group, no significant change was found at the end of the treatment, except for an increase in insulin-related metabolic parameters (Table 2).

This slight worsening of insulin AUC in the placebo group is probably due to the fact that patients were not treated for a few months before and during the study. This is a further demonstration that inositol improves metabolic patterns even after 3 months.

Comparing the two groups, significant differences were observed in BMI, serum testosterone, serum androstenedione, fasting serum insulin, insulin AUC after OGTT, and HOMA-IR (Table 2).

To examine RBC membrane status, diamide-related band 3 Tyr-P levels were determined in patients' erythrocytes. Although in the absence of diamide stimulation Tyr-P could not be detected in erythrocytes from either patients or controls (data not shown), when PCOS RBC were incubated with diamide, membranes showed higher band 3 Tyr-P levels in comparison with RBC from healthy subjects (Table 3, see footnote). Comparing RBC from PCOS patients at the two time points $\mathrm{T}_{0}$ (baseline) and $\mathrm{T}_{1}$ (following MYO treatment) shows that Tyr-P levels were significantly affected by MYO treatment (Table 3, $P<0.0001$ ). No variations were found in the placebo-treated group (Table 3).

The antioxidant condition of RBC was also evaluated by measuring changes in GSH cellular content (22). As shown in Table 3 , the decrease in GSH $(\Delta G S H)$ was

Table 2 Changes in clinical characteristics and hematochemical parameters after administration of MYO or placebo for 12 weeks in PCOS patients. Data are mean \pm s.D. of paired differences between times $T_{1}$ and $T_{0}$ for each patient. Negative sign indicates a decrease after treatment in comparison with baseline.

\begin{tabular}{|c|c|c|c|c|}
\hline Parameters & MYO group $(n=18)$ & Placebo group $(n=8)$ & $\boldsymbol{P}^{\mathrm{a}}$ & Effect size $e^{b}$ \\
\hline Weight (kg) & $-1.83 \pm 1.86^{c}$ & $+0.25 \pm 0.71$ & 0.0055 & 1.34 \\
\hline BMI $\left(\mathrm{kg} / \mathrm{m}^{2}\right)$ & $-0.69 \pm 0.69^{c}$ & $+0.09 \pm 0.27$ & 0.0058 & 1.35 \\
\hline \multicolumn{5}{|l|}{ Blood pressure $(\mathrm{mmHg})$} \\
\hline Systolic & $-3.3 \pm 5.9^{c}$ & $+1.3 \pm 6.4$ & 0.0888 & 0.79 \\
\hline Diastolic & $+0.6 \pm 2.4$ & $+2.5 \pm 4.6$ & 0.1645 & 0.62 \\
\hline Serum testosterone $(\mathrm{nmol} / \mathrm{l})$ & $-0.35 \pm 0.24^{c}$ & $-0.01 \pm 0.29$ & 0.0043 & 1.38 \\
\hline Serum androstenedione $(\mathrm{nmol} / \mathrm{l})$ & $-3.96 \pm 2.16^{c}$ & $+0.28 \pm 0.39$ & $<0.0001$ & 2.41 \\
\hline Fasting serum insulin (mU/l) & $-2.33 \pm 2.61^{\mathrm{c}}$ & $+1.00 \pm 0.76^{c}$ & 0.0018 & 1.55 \\
\hline Fasting serum glucose $(\mathrm{mmol} / \mathrm{l})$ & $-0.14 \pm 0.31$ & $+0.06 \pm 0.13$ & 0.0816 & 0.077 \\
\hline Insulin AUC (mU/l per min) & $-1668.08+1388.52^{c}$ & $+347.38+314.98^{c}$ & 0.0005 & 1.77 \\
\hline Glucose AUC (mmol// per min) & $-13.42 \pm 65.52$ & $+17.06+28.64$ & 0.2224 & 0.55 \\
\hline HOMA-IR & $-0.54+0.62^{c}$ & $+0.26+0.16^{\mathrm{c}}$ & 0.0015 & 1.57 \\
\hline
\end{tabular}

${ }^{\mathrm{a} C}$ Comparison between groups; statistical analysis with Student's $t$-test.

'The effect size was calculated post-hoc according to Cohen's $d$ formula (48), using pooled s.D.s. According to Cohen's $d$, an effect size of $0.2-0.3$ is considered a 'small' effect; around 0.5 , a 'medium' effect; and $>0.8$, a 'large' effect.

${ }^{c}$ Statistically significant variation between times $T_{1}$ and $T_{0}$ within patient groups $(P<0.05$ as significance limit); statistical analysis with paired Student's $t$-test. 
Table 3 Comparison between the parameters investigated in erythrocytes from PCOS patients in MYO and placebo groups. Data are mean \pm s.D. calculated according to the formula in the Materials and methods section. Tyr- $P$ value of diamide-treated erythrocytes from PCOS patients before $\left(T_{0}\right)$ and after $\left(T_{1}\right)$ $\mathrm{MYO} /$ placebo treatment, calculated as ratio to Tyr-P level of diamide-treated erythrocytes obtained in healthy controls (chosen as arbitrary comparison unit, experimentally determined as $0.98 \pm 0.065$, mean value \pm S.D.).

\begin{tabular}{lcrrr}
\hline Parameters & $\begin{array}{c}\text { MYO } \\
\text { group } \\
(n=18)\end{array}$ & $\begin{array}{l}\text { Placebo } \\
\text { group } \\
(n=8)\end{array}$ & $\boldsymbol{P}^{\mathrm{a}}$ & $\begin{array}{c}\text { Effect } \\
\text { size }^{\mathrm{b}}\end{array}$ \\
\hline Tyr-P & & & & \\
$\mathrm{T}_{0}$ & $2.06 \pm 0.28$ & $2.00 \pm 0.24$ & 0.5819 & 0.23 \\
$\mathrm{~T}_{1}$ & $1.36 \pm 0.30^{\mathrm{c}}$ & $2.01 \pm 0.27$ & $<0.0001$ & 2.32 \\
$\mathrm{~V}_{\mathrm{i}(\mathrm{T} y r-\mathrm{P})}$ & $0.34 \pm 0.10$ & $-0.01 \pm 0.05$ & $<0.0001$ & 4.12 \\
$\Delta \mathrm{GSH}$ & & & & \\
$\mathrm{T}_{0}$ & $0.38 \pm 0.04$ & $0.39 \pm 0.03$ & 0.5290 & 0.28 \\
$\mathrm{~T}_{1}$ & $0.23 \pm 0.06^{\mathrm{c}}$ & $0.40 \pm 0.02$ & $<0.0001$ & 3.43 \\
$\mathrm{~V}_{\mathrm{i}(\Delta \mathrm{GSH})}$ & $0.40 \pm 0.14$ & $-0.02 \pm 0.05$ & $<0.0001$ & 3.62 \\
$\Delta \mathrm{GSSP}$ & & & & \\
$\mathrm{T}_{0}$ & $0.33 \pm 0.03$ & $0.32 \pm 0.02$ & 0.3695 & 0.38 \\
$\mathrm{~T}_{1}$ & $0.17 \pm 0.04^{\mathrm{c}}$ & $0.31 \pm 0.03$ & $<0.0001$ & 3.90 \\
$\mathrm{~V}_{\mathrm{i}(\Delta \mathrm{GSSP})}$ & $0.48 \pm 0.11$ & $0.02 \pm 0.07$ & $<0.0001$ & 4.79 \\
\hline
\end{tabular}

$\Delta \mathrm{GSH}$, decrease in cytosol glutathione content after diamide treatment, expressed as $1-\mathrm{GSH}_{\text {(Diamide) }} / \mathrm{GSH}_{\text {(Basal) }} ; \Delta \mathrm{GSSP}$, increase in glutathionylated proteins after diamide treatment, expressed as the increase in glutathione released from membrane proteins or $\left(\mathrm{GSH}_{(\text {Diamide) }} / \mathrm{GSH}_{(\text {Basal })}\right)-1$ ${ }^{a} P$ value following Student's $t$-test, comparison between MYO and placebo groups.

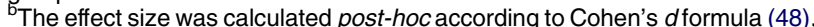
using pooled s.D.s. According to Cohen's $d$, an effect size of $0.2-0.3$ is considered a 'small' effect; around 0.5 , a 'medium' effect; and >0.8, a 'large' effect.

${ }^{c} P<0.0001$, paired Student's $t$-test, comparison between $T_{1}$ and $T_{0}$ within each group.

significantly less $(P<0.0001)$ after dietary treatment with MYO; no changes were detected in control patients.

Treatment with dietary MYO also prevented any increase in glutathionylated protein content ( $\Delta$ GSSP) in erythrocytes from PCOS patients, the variation being even lower at $\mathrm{T}_{1}$ compared with $\mathrm{T}_{0}(P<0.0001)$; conversely, no variation occurred in the placebo group (Table 3).

The variation indices $V_{i}$ (see Materials and methods section) of the three parameters of RBC membraneoxidative status allow for a direct unbiased comparison of Tyr-P, GSH, and GSSP changes occurring in the two groups of patients (Table 3). All three parameters were significantly different in MYO and placebo groups $(P<0.0001)$.

To assess interrelationships between RBC parameters and hormonal/metabolic data obtained in blood from patients at the two time points, before and after MYO treatment, a linear regression was performed and the strength of correlation was assessed by Pearson's correlation coefficient $r$ (Table 4).

The main result obtained was the significant correlation between serum testosterone and Tyr-P levels
$(P<0.05)$. The link between Tyr-P and testosterone levels before and after MYO treatment is shown in Fig. 1.

Comparison of the two regression lines, at $\mathrm{T}_{0}$ and $\mathrm{T}_{1}$, revealed a significant difference between the intercepts in MYO-treated patients $(P<0.0001)$; the slope remained the same, indicating that, although phosphorylation kinetic mechanisms were unaffected by MYO administration, they were quantitatively reduced. The same analysis applied to the placebo-treated group confirming the significant correlation between erythrocyte Tyr-P and serum testosterone levels at baseline $(P=0.0032$; data not shown $)$.

A significant correlation was also detected between insulin AUC and Tyr-P, but only before treatment (Table 4). After MYO treatment, the concomitant reduction found for Tyr-P and insulin AUC values produced a clustering of data that abolished the regression of the two variables.

\section{Discussion}

A common misconception related to PCOS is that every patient is overweight, but this is not always the case, because some of the women diagnosed with this condition are of normal weight (29). In this study, we show that IR can be present in PCOS with normal BMI, confirming that IR is not merely linked to obesity but also to a genetic or epigenetic situation $(30,31,32,33)$.

An increase in the generation of ROS by peripheral blood leucocytes in response to acute hyperglycemia has been previously demonstrated in PCOS patients (11), and the finding that physiological and pharmacological insulin infusions in vivo or in vitro revealed the oxidative effect of insulin $(32,33,34,35,36)$ raised the hypothesis of systemic redox impairment in such patients.

Our results show that this redox impairment is effectively systemic, as indicated by oxidative-related alterations in the erythrocytes of all patients. In addition, insulin AUC significantly correlated with RBC alterations in patients, rather than fasting glucose and fasting insulin or glucose AUC. The values of the last

Table 4 Correlation between hormonal/metabolic parameters and Tyr-P levels in erythrocytes before $\left(T_{0}\right)$ and after $\left(T_{1}\right)$ myo-inositol administration $(n=18)$.

\begin{tabular}{lcc}
\hline & \multicolumn{2}{c}{$\begin{array}{c}\text { Pearson's correlation } \\
\text { coefficient } r\end{array}$} \\
\cline { 2 - 3 } Tyr-P vs & $\mathrm{T}_{0}$ & $\mathrm{~T}_{1}$ \\
\hline Serum testosterone (nmol/l) & $0.5698^{*}$ & $0.4943^{*}$ \\
Serum androstenedione $(\mathrm{nmol} / \mathrm{l})$ & 0.2028 & -0.1447 \\
Fasting serum insulin $(\mathrm{mU} / \mathrm{l})$ & 0.3264 & 0.2756 \\
Fasting serum glucose $(\mathrm{mmol} / \mathrm{l})$ & -0.2193 & 0.0320 \\
Insulin AUC (mU// per min) & $0.5032^{*}$ & 0.1673 \\
Glucose AUC (mmol// per min) & -0.2076 & -0.3208 \\
HOMA-IR & 0.2968 & 0.2913 \\
\hline
\end{tabular}

${ }^{*} P<0.05$; statistical analysis of regression by ANOVA. 


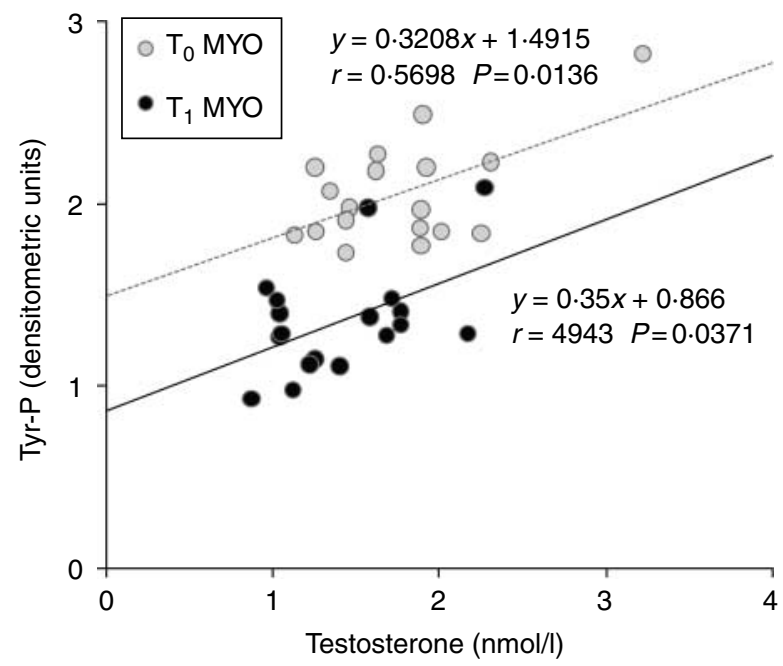

Figure 1 Linear regression between erythrocyte Tyr-P values and testosterone serum levels in MYO group before $\left(T_{0}\right)$ and after $\left(T_{1}\right)$ treatment. ANOVA indicated a significant difference $(P<0.0001)$ between intercepts of regression lines in MYO-treated patients.

three parameters were comparable with those of normal healthy women, thus corroborating the oxidative effect of insulin rather than that of hyperglycemia (Table 1).

We have recently demonstrated that enhanced ROS production in endometriotic patients is correlated with both increased diamide-induced erythrocyte band 3 Tyr-P level and high $\Delta G S H$, indicating that OS induces structural modifications of membranes (22). Diamideinduced OS in human erythrocytes causes net membrane rearrangement with band 3 aggregate formation $(16,18)$ and kinase (37) and phosphatase (38) recruitments, all leading to a triggering of band 3 Tyr-P levels (20). In PCOS, RBC are much more sensitive to diamide treatment and, consequently, band 3 Tyr-P reaches levels two or three times higher than those of controls, probably due to an altered redox system, predisposing membrane proteins to be more markedly oxidized. This was further confirmed by the observation that total cell GSH did not differ from that of healthy controls (data not shown) but that, once RBC are incubated with diamide, patients' GSH contents are far lower, with $\Delta$ GSH ranging from $0.38 \pm 0.04$ (Table 3). By contrast, when membranes were extracted and analyzed for their contents of glutathionylated proteins (GSSP), glutathionylation after diamide treatment increased drastically in PCOS RBC ( $\triangle$ GSSP, 0.33 \pm 0.03 ; Table 3 ) but not in healthy controls (data not shown). Taken together, these results show that PCOS $\mathrm{RBC}$ are subjected to OS, which induces membrane oxidative status alterations.

Interestingly, MYO treatment positively affected the oxidative status of RBC, as shown by the partial restoration of GSH contents (decreased $\Delta \mathrm{GSH}$ ) and the reduction of both band 3 Tyr-P levels and protein glutathionylation (decreased $\Delta$ GSSP). The present investigation shows that even a low dose of MYO administered for 3 months yields therapeutically appreciable effects. In fact, clinical ameliorations were achieved although the posology of $1200 \mathrm{mg}$ /day was almost one-fourth of that commonly used for MYO (39, 40 ) and similar to that for DCI (6). In addition, as an anecdotal finding, one of the patients enrolled in the MYO group continued the intake of half a dose of MYO (i.e. $600 \mathrm{mg} /$ day) after the end of the study period, and the clinical symptoms appeared again (data not shown). This would suggest that the dose used in our study may represent the lowest range of a posology aimed at obtaining an appreciable effect in PCOS patients.

This study has also evaluated the hormonal and metabolic status of PCOS patients, and compared values at $\mathrm{T}_{0}$ and $\mathrm{T}_{1}$, to analyze the effect of MYO treatment on clinical parameters.

Our findings confirm that oral MYO administration significantly decreases testosterone, androstenedione, fasting serum insulin, insulin AUC levels, and HOMA-IR.

Two different pathways may depend on MYO: phosphoinositide-related signal transduction and insulin-related metabolic response. In the first pathway, MYO, synthesized from glucose 6-phosphate, carried into the cell from plasma or obtained by a recycling of intracellular inositol 1,4,5-triphosphate, is incorporated into activated phosphatidic acid to constitute membrane phosphatidylinositols (PI) $(40,41,42,43)$. In the second pathway, either MYO or its epimerase-converted (39) chiro isoform DCI $(44,45)$ constitutes IPGs $(4)$, which are released from cell membrane glycosylphosphatidylinositols in response to insulin. Once released, IPGs can affect any tissues and cells implicated in insulin action (45), thus potentiating insulin effects (46).

The significant reduction of Tyr-P levels in RBC found after MYO treatment and the reduction in insulin AUC confirm that this kind of therapy can improve IR, one of the typical features of PCOS (10). In fact, dietary MYO uptake can affect membrane composition both directly, by increasing intracellular PI contents and/or PI-related pathways, and indirectly, by modulating the OS induced by the inappropriate hyperinsulinemic response linked to IR.

Another novel finding is the significant correlation between Tyr-P and testosterone levels at $\mathrm{T}_{0}$ and, more interestingly, at $\mathrm{T}_{1}$ (Table 4), as shown by a comparison of the two regression lines (Fig. 1). The significant difference between the intercepts of the two lines in MYO-treated patients $(P<0.0001)$ suggests that alteration of the phosphorylation process of erythrocyte band 3 is mediated by higher testosterone levels in serum. We hypothesize that administration of MYO (but not of placebo) improves band 3 Tyr-P (and related $\Delta G S H$ and $\Delta$ GSSP) through a decrease in testosterone levels, thus supporting the MYO-related insulin messenger pathway.

In conclusion, PCOS patients suffer from a systemic inflammatory status, as shown by the increased erythrocyte response to diamide-induced OS, with 
increases in Tyr-P level, $\Delta$ GSH, and $\Delta$ GSSP parameters, which significantly correlate with testosterone levels. The use of MYO, even at $1200 \mathrm{mg} /$ day, reduced inflammatory status and IR-related hyperinsulinemia (10, 47).

Further studies investigating both the intracellular mechanism triggered by inositol and the extracellular inositol-related IPG pathways are warranted to better define how MYO could improve insulin response and oxidative status in patients with PCOS. The Tyr-P process could be very useful in monitoring patients' conditions and in choosing an adequate therapy for IR. In addition, MYO could be proposed as an alternative to metformin treatment because the former can affect insulin target tissues and cells and potentiate insulin effects without the side effects of metformin.

\section{Declaration of interest}

The authors declare that there is no conflict of interest that could be perceived as prejudicing the impartiality of the research reported.

\section{Funding}

This work was supported by the Italian Ministero dell'Università e della Ricerca Scientifica e Tecnologica (MURST).

\section{References}

1 Diamanti-Kandarakis E, Kouli CR, Bergiele AT, Filandra FA, Tsianateli TC, Spina GG, Zapanti ED \& Bartzis MI. A survey of the polycystic ovary syndrome in the Greek island of Lesbos: hormonal and metabolic profile. Journal of Clinical Endocrinology and Metabolism 199984 4006-4011. (doi:10.1210/jc.84.11. 4006)

2 Nestler JE. Role of hyperinsulinemia in the pathogenesis of the polycystic ovary syndrome, and its clinical implications. Seminars in Reproductive Endocrinology 199715 111-122. (doi:10.1055/s2007-1016294)

3 Apridonidze T, Essah PA, Iuorno MJ \& Nestler JE. Prevalence and characteristics of the metabolic syndrome in women with polycystic ovary syndrome. Journal of Clinical Endocrinology and Metabolism 200590 1929-1935. (doi:10.1210/jc.20041045)

4 Romero G \& Larner J. Insulin mediators and the mechanism of insulin action. Advances in Pharmacology 199324 21-50. (doi:10. 1016/S1054-3589(08)60932-1)

5 Saltiel AR. Second messengers of insulin action. Diabetes Care 199013 244-256. (doi:10.2337/diacare.13.3.244)

6 Nestler JE, Jakubowicz DJ, Reamer P, Gunn RD \& Allan G. Ovulatory and metabolic effects of D-chiro-inositol in the polycystic ovary syndrome. New England Journal of Medicine $1999 \mathbf{3 4 0}$ 1314-1320. (doi:10.1056/NEJM199904293401703)

7 Iuorno MJ, Jakubowicz DJ, Baillargeon JP, Dillon P, Gunn RD, Allan G \& Nestler JE. Effects of D-chiro-inositol in lean women with the polycystic ovary syndrome. Endocrine Practice 20028 $417-423$.

8 Gerli S, Mignosa M \& Di Renzo GC. Effects of inositol on ovarian function and metabolic factors in women with PCOS: a randomized double blind placebo-controlled trial. European Review for Medical and Pharmacological Sciences 2003 7 151-159.

9 Baillargeon JP, Diamanti-Kandarakis E, Ostlund RE Jr, Apridonidze T, Iuorno MJ \& Nestler JE. Altered D-chiro-inositol urinary clearance in women with polycystic ovary syndrome. Diabetes Care 200629 300-305. (doi:10.2337/diacare.29.02.06. dc05-1070)

10 Baillargeon JP, Nestler JE, Ostlund RE, Apridonidze T \& DiamantiKandarakis E. Greek hyperinsulinemic women, with or without polycystic ovary syndrome, display altered inositols metabolism. Human Reproduction 200823 1439-1446. (doi:10.1093/humrep/den097)

11 Gonzàlez F, Rote NS, Minium J \& Kirwan JP. Reactive oxygen species-induced oxidative stress in the development of insulin resistance and hyperandrogenism in polycystic ovary syndrome. Journal of Clinical Endocrinology and Metabolism 200691 336-340. (doi:10.1210/jc.2005-1696)

12 Victor VM, Rocha M, Bañuls C, Sanchez-Serrano M, Sola E, Gomez M \& Hernandez-Mijares A. Mitochondrial complex I impairment in leukocytes from polycystic ovary syndrome patients with insulin resistance. Journal of Clinical Endocrinology and Metabolism 200994 3505-3512. (doi:10.1210/jc.2009-0466)

13 Sabuncu T, Vural H, Harma M \& Harma M. Oxidative stress in polycystic ovary syndrome and its contribution to the risk of cardiovascular disease. Clinical Biochemistry 200134 407-413. (doi:10.1016/S0009-9120(01)00245-4)

14 Stadtman ER \& Levine RL. Protein oxidation. Annals of the New York Academy of Sciences 2000899 191-208. (doi:10.1111/j. 1749-6632.2000.tb06187.x)

15 Pacifici RE \& Davies KJ. Protein degradation as an index of oxidative stress. Methods in Enzymology 1990186 485-502. (doi:10.1016/0076-6879(90)86143-J)

16 Bordin L, Quartesan S, Zen F, Vianello F \& Clari G. Band 3 Tyr-phosphorylation in human erythrocytes from non-pregnant and pregnant women. Biochimica et Biophysica Acta $2006 \mathbf{1 7 5 8}$ 611-619. (doi:10.1016/j.bbamem.2006.03.009)

17 Fiore C, Bordin L, Pellati D, Armanini D \& Clari G. Effect of glycyrrhetinic acid on membrane band 3 in human erythrocytes. Archives of Biochemistry and Biophysics 2008 479 46-51. (doi:10. 1016/j.abb.2008.08.011)

18 Bordin L, Fiore C, Zen F, Coleman MD, Ragazzi E \& Clari G. Dapsone hydroxylamine induces premature removal of human erythrocytes by membrane reorganization and antibody binding. British Journal of Pharmacology 2010161 1186-1199. (doi:10.1111/j. 1476-5381.2010.00962.x)

19 Salvemini D \& Cuzzocrea S. Oxidative stress in septic shock and disseminated intravascular coagulation. Free Radical Biology $\mathcal{E}$ Medicine 200233 1173-1185. (doi:10.1016/S08915849(02)00961-9)

20 Bordin L, Ion-Popa F, Brunati AM, Clari G \& Low PS. Effectorinduced Syk-mediated phosphorylation in human erythrocytes. Biochimica et Biophysica Acta 20051745 20-28. (doi:10.1016/j. bbamcr.2004.12.010)

21 Bordin L, Zen F, Ion-Popa F, Barbetta M, Baggio B \& Clari G. Band 3 Tyr-phosphorylation in normal and glucose-6-phosphate dehydrogenase-deficient human erythrocytes. Molecular Membrane Biology 200522 411-420. (doi:10.1080/09687860 500233679)

22 Bordin L, Fiore C, Donà G, Andrisani A, Ambrosini G, Faggian D, Plebani M, Clari G \& Armanini D. Evaluation of erythrocyte band 3 phosphotyrosine level, glutathione content, CA-125, and human epididymal secretory protein $\mathrm{E} 4$ as combined parameters in endometriosis. Fertility and Sterility 201094 1616-1621. (doi:10.1016/j.fertnstert.2009.10.038)

23 Clements RS Jr \& Darnell B. Myo-inositol content of common foods: development of a high-myo-inositol diet. American Journal of Clinical Nutrition 198033 1954-1967.

24 Horbowicz M, Brenac P \& Obendorf RL. Fagopyritol B1, O-alphaD-galactopyranosyl-( $1 \rightarrow 2)$-D-chiro-inositol, a galactosyl cyclitol in maturing buckwheat seeds associated with desiccation tolerance. Planta 1998205 1-11. (doi:10.1007/s004250050290)

25 Rotterdam ESHRE/ASRM-Sponsored PCOS Consensus Workshop Group. Revised 2003 consensus on diagnostic criteria and longterm health risks related to polycystic ovary syndrome. Fertility and Sterility $2004 \mathbf{8 1} 19-25$. 
26 Matthews DR, Hosker JP, Rudenski AS, Naylor BA, Treacher DF \& Turner RC. Homeostasis model assessment: insulin resistance and beta-cell function from fasting plasma glucose and insulin concentrations in man. Diabetologia 198528 412-419. (doi:10. 1007/BF00280883)

27 Tietze F. Enzymic method for quantitative determination of nanogram amounts of total and oxidized glutathione: applications to mammalian blood and other tissues. Analytical Biochemistry 196927 502-522. (doi:10.1016/0003-2697(69)90064-5)

28 Di Simplicio P, Cacace MG, Lusini L, Giannerini F, Giustarini D \& Rossi R. Role of protein -SH groups in redox homeostasis - the erythrocyte as a model system. Archives of Biochemistry and Biophysics 1998355 145-152. (doi:10.1006/abbi.1998.0694)

29 Economou F, Xyrafis X, Livadas S, Androulakis II, Argyrakopoulou G, Christakou CD, Kandaraki E, Palioura E \& Diamanti-Kandarakis E. In overweight/obese but not in normalweight women, polycystic ovary syndrome is associated with elevated liver enzymes compared to controls. Hormones 20098 199-206.

30 Takeuchi T, Tsutsumi O \& Taketani Y. Abnormal response of insulin to glucose loading and assessment of insulin resistance in non-obese patients with polycystic ovary syndrome. Gynecological Endocrinology $2008 \quad 24 \quad 385-391 . \quad$ (doi:10.1080/095135 90802173584)

31 Dunaif A, Segal KR, Futterweit W \& Dobrjansky A. Profound peripheral insulin resistance, independent of obesity, in polycystic ovary syndrome. Diabetes 198938 1165-1174. (doi:10.2337/ diabetes.38.9.1165)

32 Diamanti-Kandarakis E, Alexandraki K, Bergiele A, Kandarakis H, Mastorakos G \& Aessopos A. Presence of metabolic risk factors in non-obese PCOS sisters: evidence of heritability of insulin resistance. Journal of Endocrinological Investigation 200427 931-936.

33 Macut D, Simic T, Lissounov A, Pljesa-Ercegovac M, Bozic I, Djukic T, Bjekic-Macut J, Matic M, Petakov M, Suvakov S, Damjanovic S \& Savic-Radojevic A. Insulin resistance in non-obese women with polycystic ovary syndrome: relation to byproducts of oxidative stress. Experimental and Clinical Endocrinology \& Diabetes 2011119 451-455. (doi:10.1055/s0031-1279740)

34 Kriger-Brauer HI \& Kather H. Human fat cells possess a plasma membrane-bound $\mathrm{H}_{2} \mathrm{O}_{2}$-generating system that is activated by insulin via a mechanism bypassing the receptor kinase. Journal of Clinical Investigation $1992 \quad 89$ 1006-1013. (doi:10.1172/ JCI115641)

35 Rifici VA, Schneider SH \& Khachadurian AK. Stimulation of lowdensity lipoprotein oxidation by insulin and insulin like growth factor I. Atherosclerosis 1994107 99-108. (doi:10.1016/00219150(94)90145-7)

36 Galvan AQ, Muscelli E, Catalano C, Natali A, Sanna G, Masoni A, Bernardini B, Barsacchi R \& Ferrannini E. Insulin decreases circulating vitamin E levels in humans. Metabolism: Clinical and Experimental $1996 \quad 45$ 998-1003. (doi:10.1016/S00260495(96)90270-X)
37 Brunati AM, Bordin L, Clari G, James P, Quadroni M, Baritono E, Pinna LA \& Donella-Deana A. Sequential phosphorylation of protein band 3 by Syk and Lyn tyrosine kinases in intact human erythrocytes: identification of primary and secondary phosphorylation sites. Blood 200096 1550-1557.

38 Bordin L, Brunati AM, Donella-Deana A, Baggio B, Toninello A \& Clari G. Band 3 is an anchor protein and a target for SHP-2 tyrosine phosphatase in human erythrocytes. Blood $2002 \mathbf{1 0 0}$ 276-282. (doi:10.1182/blood.V100.1.276)

39 Carlomagno G, Unfer V \& Roseff S. The D-chiro-inositol paradox in the ovary. Fertility and Sterility 201195 2515-2516. (doi:10. 1016/j.fertnstert.2011.05.027)

40 Carlomagno G \& Unfer V. Inositol safety: clinical evidences. European Review for Medical and Pharmacological Sciences 201115 931-936.

41 Di Daniel E, Kew JN \& Maycox PR. Investigation of the H(+ )-myoinositol transporter (HMIT) as a neuronal regulator of phosphoinositide signalling. Biochemical Society Transactions 200937 1139-1143. (doi:10.1042/BST0371139)

42 Larner J. Mediators of postreceptor action of insulin. American Journal of Medicine $1983 \mathbf{7 4} 38-51$. (doi:10.1016/00029343(83)90653-8)

43 Larner J. Insulin-signaling mechanisms. Lessons from the old testament of glycogen metabolism and the new testament of molecular biology. Diabetes 198837 262-275.

44 Larner J, Huang LC, Suzuki S, Tang G, Zhang C, Schwartz CFW, Romero G, Luttrell L \& Kennington AS. Insulin mediators and the control of pyruvate dehyrogenase complex. Annals of the New York Academy of Sciences 1989573 297-305. (doi:10.1111/j.17496632.1989.tb15006.x)

45 Jones DR \& Varela-Nieto I. The role of glycosyl-phosphatidylinositol in signal transduction. International Journal of Biochemistry $\mathcal{E}$ Cell Biology 199830 313-326. (doi:10.1016/S13572725(97)00144-1)

46 Cheang KI, Baillargeon JP, Essah PA, Ostlund RE Jr, Apridonize T, Islam L \& Nestler JE. Insulin-stimulated release of D-chiro-inositolcontaining inositolphosphoglycan mediator correlates with insulin sensitivity in women with polycystic ovary syndrome. Metabolism: Clinical and Experimental 200810 1390-1397. (doi:10.1016/j. metabol.2008.05.008)

47 Papaleo E, Unfer V, Baillargeon JP \& Chiu TT. Contribution of myoinositol to reproduction. European Journal of Obstetrics, Gynecology, and Reproductive Biology 2009147 120-123. (doi:10.1016/j. ejogrb.2009.09.008)

48 Cohen J. Statistical Power Analysis for the Behavioral Sciences. New York: Academic Press, 1977.

Received 29 September 2011

Revised version received 23 December 2011

Accepted 5 January 2012 